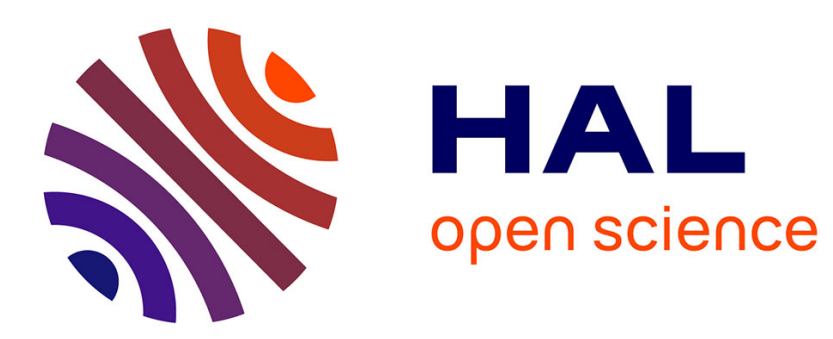

\title{
Latitudinal distribution of solar wind as deduced from Lyman alpha measurements: An improved method
}

\author{
T. Summanen, Rosine Lallement, Jean-Loup Bertaux, E. Kyrölä
}

\section{To cite this version:}

T. Summanen, Rosine Lallement, Jean-Loup Bertaux, E. Kyrölä. Latitudinal distribution of solar wind as deduced from Lyman alpha measurements: An improved method. Journal of Geophysical Research Space Physics, 1993, 98 (A8), pp.13215-13224. 10.1029/93JA00144 . insu-02889807

\section{HAL Id: insu-02889807 https://hal-insu.archives-ouvertes.fr/insu-02889807}

Submitted on 3 Feb 2021

HAL is a multi-disciplinary open access archive for the deposit and dissemination of scientific research documents, whether they are published or not. The documents may come from teaching and research institutions in France or abroad, or from public or private research centers.
L'archive ouverte pluridisciplinaire HAL, est destinée au dépôt et à la diffusion de documents scientifiques de niveau recherche, publiés ou non, émanant des établissements d'enseignement et de recherche français ou étrangers, des laboratoires publics ou privés. 


\title{
Latitudinal Distribution of Solar Wind as Deduced From Lyman Alpha Measurements: An Improved Method
}

\author{
T. SUMMANEN
}

Finnish Meteorological Institute, Helsinki, Finland

R. LALLEMENT AND J. L. BERTAUX

Service d'Aeronomie du Centre National de la Recherche Scientifique, Verridres-le-Buisson, France

\section{E. KYRÖLÄ}

Finnish Meteorological Institute, Helsinki, Finland

\begin{abstract}
In this work we examine the possibility of deducing the latitudinal distribution of the solar ionization rate using Prognoz 6 Lyman alpha data in a more general and flexible way than previously examined. Using socalled hot model for the hydrogen distribution and the optically thin model for the resonance scattering, theoretical Lyman alpha intensity for the interstellar hydrogen is calculated and compared with the intensity data measured by Prognoz 6. Varying the latitudinal dependence of the ionization rate, the distributions, which produce the best fit with the data, are analyzed for four different measuring sessions. As a result, we get four ionization rate distributions that have two common features. The ionization rate is enhanced near the solar equator, and large broad plateaus exist around heliographic latitudes $\pm 30^{\circ}$ to $\pm 70^{\circ}$. The latitudinal distribution of the average ionization rate about the solar minimum deviates clearly from the spherically symmetric and sinusoidally (harmonically) with the latitude-varying models used so far. The growth of the solar wind mass flux from the solar polar areas toward the equator corresponds to the earlier results concluded from Lyman alpha measurements. The method used in this work allows a higher latitudinal resolution of the ionization rates. However, there are several uncertainties both in the simulations and in the measurements. The exclusion of time-dependent effects as well as multiple scattering requires that the results be considered only suggestive.
\end{abstract}

\section{INTRODUCTION}

Studies of coronal holes in the 1970s have led to the development of a model for the spatial structure of the solar wind. The solar polar regions develop into large coronal holes during the declining phase of the solar cycle, and the holes begin to expand toward the equator. The polar holes attain their greatest size at the minimum phase. At the maximum phase they disappear. The temporal variations of coronal holes suggested latitudinal changes in the solar wind proton flux, which is the main ionizing effect of the interplanetary hydrogen.

At the same time, the interplanetary scintillation (IPS) observations showed that the average solar wind speed increases from the equator toward the polar regions [Coles and Rickett, 1976]. It was discovered that the major sources of the low-speed flow are distributed along the neutral lines through the whole solar cycle. During low solar activity the neutral line lies almost parallel to the solar equator. The high-speed flows seem to originate then from the polar coronal holes. An extensive review concerning solar wind speed structure observed using the IPS method from 1973 to 1987 has been published recently by Kojima and Kakinuma [1990].

Until 1975, spherically-symmetric solar radiation fields for both corpuscular and electromagnetic radiation were assumed while modeling of the neutral hydrogen distribution. Then Joselyn and Holzer [1975] studied theoretically the effect of

\section{Copyright 1993 by the American Geophysical Union.}

Paper number 93JA00144. 0148-0227/93/93JA-00144\$05.00 anisotropic solar wind on the Lyman $\alpha$ sky background. They assumed that the solar wind proton flux would increase near the polar regions and concluded that latitudinal variations would cause a significant change in the Lyman $\alpha$ intensity. Kumar and Broadfoot [1978, 1979] studied the solar wind's latitudinal structure by comparing the Mariner 10 Lyman $\alpha$ observations and the theoretically calculated intensities. According to their studies the harmonic ionization rate clearly better fits the data than the spherically symmetric model. The same latitudinal structure has been used also by many other authors [Witt et al., 1979, 1981; Isenberg and Levy, 1978; Lallement et al., $1985 b]$.

The main reason to use the harmonic model is that it allows an analytical evaluation of the hydrogen atom loss rate. However, the latitudinal variations of the ionization rate seem to be so fast that the slowly varying harmonic model cannot model them. The independent studies of the solar wind velocity seem to support this idea [Kojima and Kakinuma, 1990]. In the present work a different approach has been taken. The demand for analytical expressions has been ignored, and the ionization rates have been computed numerically.

A computer code based on these ideas has been used to produce simulated measurements, which have been compared with the Lyman $\alpha$ data measured by Prognoz 6. The measuring sessions used in this work are the same as those used by Lallement et al. [1985b]. As a result we have obtained an ionization rate distribution, which produced the best fit with data, for each data session. Thus some features of the latitudinal dependence of the solar wind ionization rate at $1 \mathrm{AU}$ can be predicted.

In the following sections the hydrogen distribution model as well as the optically thin scattering model are briefly reviewed. 
Later in the paper the numerically computed model results are presented. Also, the latitudinal variation of the solar wind flux is estimated using the solar wind velocity model by Zhao and Hundhausen [1981]. The conclusions are presented and discussed in the final section.

In this work the problems concerning the heliopause are ignored. Thus the values used as the very local interstellar matter parameters should be understood to characterize the wind right after the heliopause, even though the expression "value at infinity" is used. The interested reader is referred to the comprehensive reviews concerning the heliosphere [Axford, 1972, 1985; Lee, 1988; Holzer, 1989; Baranov, 1990; Suess, 1990].

\section{LYMAN $\alpha$ INTENSITY SIMULATIONS}

\subsection{Hydrogen Distribution Models}

The equation of continuity in the context of the neutral interstellar gas flow has been used by many authors [e.g., Fahr, 1968; Blum and Fahr, 1970; Axford, 1972; Johnson, 1972]. The total density $\mathbf{n}(\mathbf{r})$ is obtained by integrating over the velocity distribution at infinity and summing the contributions of the two trajectories leading to the same space point $r$ :

$$
\begin{gathered}
n(r)=\left(\frac{m_{h}}{2 \pi k T_{\infty}}\right)^{3 / 2} \\
\sum_{j=1,2} \frac{n_{0} p_{j}{ }^{2} L \exp \left(\frac{-m_{h}}{2 k T_{\infty}} v_{t h}{ }^{2}\right) d^{3} v_{\infty}}{r|\sin \theta| \sqrt{r^{2} \sin ^{2} \theta+4 r(1-\cos \theta) / C}}
\end{gathered}
$$

where $\mathbf{n}$ is the atomic hydrogen density, the subscript $\mathbf{j}$ specifies the trajectory, $p_{j}$ is an impact parameter, $\theta$ is the polar angle from the upwind axis (axis of symmetry) to the point $r$ and $n_{0}$ is the hydrogen density at infinity. The abbreviation $C=v_{\infty}{ }^{2} / \gamma M_{s}(1-\mu)$, where $v_{\infty}$ is the velocity of an atom at infinity, $\gamma$ is the gravitational constant, $M_{s}$ is the mass of the Sun, and $\mu$ is the ratio between the force exerted on an atom by radiation and the gravitational force. A neutral atom has the velocity $v_{\infty}=v_{0}+v_{\text {th }}$ at infinity, where $v_{\text {th }}$ is the thermal velocity and $v_{0}$ is the bulk velocity. The thermal velocity distribution is assumed to be Maxwellian. Other parameters are conventional.

The loss effects are taken into account by introducing the loss factor $L_{j}$ :

$$
L_{j}=\exp \left(-\left|\int_{\theta_{j}}^{\theta} \frac{\beta\left(\lambda, r^{\prime}\right) r^{\prime 2}}{v_{\infty} p_{j}} d \theta^{\prime}\right|\right)
$$

where $\lambda=\lambda\left(\theta^{\prime}\right)$ is the heliographic latitude and $\beta(\lambda, r)$ is the heliographic latitudinal distribution of the ionization rate at the distance I from the Sun. The exponential factor is the ionization probability of an atom between infinity and the point $(r, \theta)$. The lower limit of the integration refers to the position of an atom at infinity and $\theta^{\prime}$ is the angle between the axis of symmetry and the point on the trajectory of an atom. In the case of a direct trajectory, $\theta_{j}$ is 0 , while for the indirect trajectory, it is $2 \pi$.

Through this work it is assumed that on their way through the solar system the neutral atoms are unaffected by any other particles except the solar wind protons in the charge transfer collisions. Ionizing collisions with other species as well as elastic collisions are neglected. The ionization rate $\beta(\lambda)$ is then given by

$$
\begin{gathered}
\beta(\lambda, r)=\beta_{c e}(\lambda, r)+\beta_{p h}(\lambda, r) \\
=n_{p r}(\lambda, r)\left|v_{r e l}(\lambda, r)\right| \sigma_{c e}\left(\left|v_{r e l}(\lambda, r)\right|\right)+\beta_{p h}(\lambda, r)
\end{gathered}
$$

where $\beta_{c e}$ is the charge exchange ionization rate, $\beta_{p h}$ is the photoionization rate, $n_{\mathrm{pr}}$ is the solar wind proton density, $v_{\text {rel }}$ is the relative velocity of solar wind protons with respect to hydrogen atoms $\left(\approx v_{p r}\right.$, the solar wind proton velocity), and $\sigma_{c e}$ is the cross section for the charge exchange collisions between solar wind protons and hydrogen atoms. We assume that $\beta(\lambda, r)$ $=\beta_{e}(\lambda) r_{e}{ }^{2} / r^{2}$, where $r_{e}$ is $1 A U$ and $\beta_{e}(\lambda)$ is the ionization rate at $1 \mathrm{AU}$. The assumption is valid for the photoionization (solar EUV flux is considered isotropic), but in the case of charge exchange processes, its validity can be questioned. However, measurements have shown that outside the Earth's orbit (1 AU) the solar wind flows approximately radially outward and its velocity is independent of the distance from the Sun [Smith and Barnes, 1983].

Two models used so far for the latitudinal dependence of the ionization rate are a spherical and a harmonic model. In a spherically symmetric (isotropic) model $\beta_{e}(\lambda)=\beta_{e}=$ const, and the loss factor simplifies to

$$
L_{j}=\exp \left(-\frac{\beta_{e} r_{e}{ }^{2}}{v_{\infty} P_{j}}\left|\theta-\theta_{j}\right|\right)
$$

The harmonic model is stated as

$$
\beta_{e}(\lambda)=\beta_{e}\left(1-A \sin ^{2} \lambda\right)
$$

where $A$ is a constant. Usually $A$ is assumed to be positive, which means the decrease of the ionization rate from the equator toward the poles. However, in an earlier paper by Joselyn and Holzer the increasing ionization rate was also studied. In later studies [e.g., Lallement et al., 1985b] it was clearly shown that the decreasing ionization rate fits the data remarkably well. This model has been used by Kumar and Broadfoot [1979] and Witt et al. [1979] when analyzing Mariner 10 Lyman $\alpha$ measurements and Lallement et al. [1985b] in Prognoz studies. The harmonic model clearly better fits the intensity measurements than the spherical model as has been shown by Lallement et al. [1985b].

In the two models introduced above the integration in the loss factor (2) could be performed analytically. In this work a different approach is used: an arbitrary latitudinal dependence is used for the ionization rate. To calculate the integral in (2), we have to know the dependence between the solar latitude $\lambda$ and the angle $\theta^{\prime}$ in the orbital plane of a hydrogen atom. The coordinate systems as well as coordinate transformations between different systems are discussed in detail by Summanen [1992]. The loss factor (2) can be written in the form 


$$
L_{j}=\exp \left(-\frac{r_{e}^{2}}{v_{\infty} P_{j}}\left|\int_{\theta_{j}}^{\theta} \beta_{e}\left(\lambda\left(\theta^{\prime}\right)\right) d \theta^{\prime}\right|\right)
$$

Thus any distribution for the ionization rate can be introduced and the integration can be evaluated numerically.

\subsection{Lyman $\alpha$ Radiation}

The solar Lyman $\alpha$ radiation is scattered resonantly by neutral hydrogen distribution. When deriving the specific intensity of Lyman $\alpha$ radiation, the material is assumed to be optically thin. Thus pure single scattering is assumed, and the absorption by intervening hydrogen is neglected.

The solar Lyman $\alpha$ line is taken to be flat. The assumption is reasonable, since the half width of the solar Lyman $\alpha$ line is about $0.5 \AA$ [e.g., Lemaire et al., 1978], and the relative motions of the atoms cause the Doppler shift less than $0.1 \AA$ (corresponds to the velocity $24 \mathrm{~km} / \mathrm{s}$ ). Thus the solar flux in photons $\mathrm{m}^{-2} \mathrm{~s}^{-1} \AA^{-1}$ is given by

$$
F\left(\lambda_{L}, r\right)=F_{0, e} \frac{r_{e}{ }^{2}}{r^{2}}
$$

where $\mathrm{F}_{0, e}$ is the solar flux at the center of the line at $1 \mathrm{AU}$. The total intensity in photons in $\mathrm{m}^{-2} \mathrm{~s}^{-1} \mathrm{sr}^{-1}$ is obtained by integrating along the line of sight:

$$
\begin{gathered}
\int I\left(\lambda_{\mathrm{L}}^{\prime}\right) \mathrm{d} \lambda_{\mathrm{L}}{ }^{\prime}= \\
\frac{\mathrm{e}^{2} \mathrm{f}}{4 \mathrm{e}_{0} \mathrm{~m}_{\mathrm{el}} \mathrm{c}} \frac{\lambda_{\mathrm{L}, 0^{2}}}{\mathrm{c}} \mathrm{F}_{0, \mathrm{e}} \int_{0}^{\infty} \frac{\mathrm{p}(\vartheta)}{4 \pi} \mathrm{n}(\mathrm{r}) \frac{\mathrm{r}_{\mathrm{e}}{ }^{2}}{\mathrm{r}^{2}} \mathrm{ds}
\end{gathered}
$$

where $\lambda_{L}^{\prime}$ is the wavelength of the measured intensity, $I\left(\lambda_{L}^{\prime}\right)$ is the specific intensity (or spectral radiance or surface brightness) in photons $\mathrm{m}^{-2} \mathrm{~s}^{-1} \AA^{-1} \mathrm{sr}^{-1}, \mathrm{p}(\vartheta)$ is the scattering phase function and other quantities are conventional.

The oscillator strength $f$ for the absorption $1 \mathrm{~s} \rightarrow 2 p$ of the hydrogen atom is 0.416 [Weissbluth, 1978, p. 516]. The scattering phase factor $\mathrm{p}(\vartheta)$ for the transition of the hydrogen atom from the ground state $1 \mathrm{~s}{ }^{2} S_{1 / 2}$ to the states $2 p^{2} P_{1 / 2}$ and ${ }^{2}{ }^{2} \mathrm{P}_{3 / 2}$ and back has been derived by Brandt and Chamberlain [1959]:

$$
p(\vartheta)=1+\frac{1}{4}\left(\frac{2}{3}-\sin ^{2} \vartheta\right)
$$

For details concerning the determination of $p(\vartheta)$, see Chamberlain and Hunten [1987] and Chamberlain [1990] and references therein. The phase function $p / 4 \pi$ is normalized to unity.

Equation (8) gives the total intensity in photons $\mathrm{m}^{-2} \mathrm{~s}^{-1} \mathrm{sr}^{-1}$. A generally used unit for intensity is Rayleigh which is $10^{6} /(4 \pi)$ photons $\mathrm{cm}^{-2} \mathrm{~s}^{-1} \mathrm{sr}^{-1}$.
The solar flux, when $\mu=1$, is assumed to be

$$
F_{0, e}=\frac{g_{e}}{\sigma_{0}}=3.3 \times 10^{11} \text { photons } \mathrm{m}^{-2} \mathrm{~s}^{-1} \AA^{-1}
$$

where $g_{e}$ is the number of photons, which collide with a hydrogen atom per second at the distance $1 \mathrm{AU}$ from the Sun and $\left.\sigma_{0}=\mathrm{e}^{2} \mathrm{f} \lambda^{2} /(4 \pi) \mathrm{mc}^{2}\right)$. For any other value of $\mu$, the solar flux is given by

$$
F_{0, e}(\mu)=\mu F_{0, e}
$$

Lyman $\alpha$ intensity data used in this work were measured by photometers onboard the Prognoz 6 satellite. The observations were performed right after the solar minimum (1976): from September 1977 to January 1978. Thus neutral hydrogen was probed right after the time period, when it had been exposed to the quiet solar wind and when the polar coronal holes had their largest size. Prognoz mission, observation characteristics and four sessions S1, S2, S3, and S4 used in this work (see Figure 1) are explained by Bertaux et al. [1985] and Lallement et al. $[1984,1985 b]$. The method to separate the remaining geocoronal contamination as well as technical details and the gaps in data due to stars and geocorona are explained in the paper by Bertaux et al. [1985], too.

\subsection{Discrete Model}

The harmonic model introduced above was used as a starting point in simulations. In Prognoz 5 and 6 studies the best value for the asymmetry constant $A$ was found to be 0.40 , and the best value for the ionization rate was $5.0 \times 10^{-7} \mathrm{~s}^{-1}$ [see Lallement et al., 1985b]. Thus the ionization rate was

$$
\beta_{e}(\lambda)=5.0 \times 10^{-7} \mathrm{~s}^{-1}\left(1-0.4 \sin ^{2} \lambda\right)
$$

In this work this function was discretized by fixing the rate at discrete heliographic latitudes:

$$
\beta_{e, i}=\beta_{e}\left(\lambda_{i}\right), i=1, \ldots, M
$$
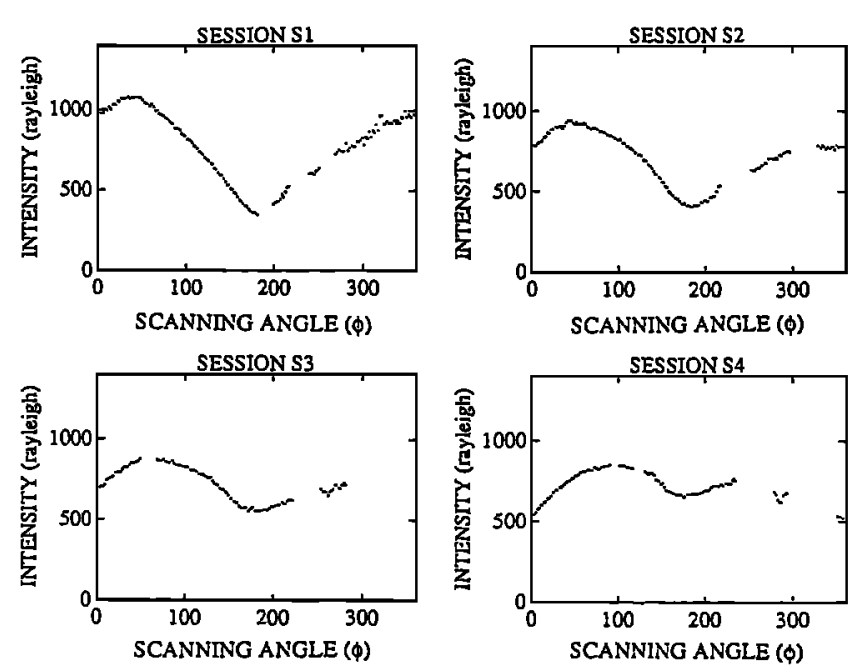

Fig. 1. The intensity data of measuring sessions S1, S2, S3, and $S 4$ as a function of the scanning angle $\phi$. 
where $M$ is the number of the points, where the ionization rate is given. The first value of the ionization rate was calculated from (12) using 19 different latitude values every $10^{\circ}$, that is, from $-90^{\circ}$ to $90^{\circ}$. The effective number of the discrete points was 10 , because the symmetric behavior in regard to the equator was assumed. Thus 10 discrete ionization rates are used as parameters instead of two parameters used in the harmonic model and one in the spherical model. Between the discrete points the ionization rate was calculated using linear interpolation. The first discrete model is shown in Figure 2.

The first step of the calculation was to introduce this discrete model in the loss factor in (6). Then the hydrogen density could be derived from (1) and finally, when the density was known, the total intensity could be calculated from (8). In the course of simulation, it was required that the ionization rate would grow or stay constant from the polar regions toward the equator. A preliminary value of the density $\left(0.04 \mathrm{~cm}^{-3}\right)$ at infinity was used. The final density value was calculated separately for each session after the scaling of the intensity data in order to produce the best fit for each ionization rate model (see Table 1). The density at infinity varies from 0.029 to $0.037 \mathrm{~cm}^{-3}$.

The values of other parameters used in all simulations are shown in Table 2. The set of parameters is deduced from Prognoz 5 and 6 measurements [Bertaux et al., 1985], except the latitude of the interstellar wind with respect to the ecliptic plane $\left(w_{1 a t}\right)$. The values $w_{10 n}$ and $w_{1 \text { at }}$ are those deduced by Dalaudier et al. [1984] in their studies of the interstellar helium. The reason we use the value $6^{\circ}$ instead of $7.5^{\circ}$ [Lallement et al., 1985a] is that it was found that $6^{\circ}$ fits the data better. In fact, the increase of latitude seemed to move the ionization maximum away from the equator.

The simulated intensity was calculated for $\mathbf{3 0}$ different lines of sight in each session. The lines of sight chosen were separated by $12^{\circ}$ so that angles $\phi[$ [Bertaux et al., 1985, Figure 1] were $0^{\circ}, 12^{\circ}, 24^{\circ}, \ldots, 336^{\circ}$, and $348^{\circ}$. The angle $\phi$ is the angle between the negative Earth's velocity vector and the line of sight. These 30 simulated intensity values were compared to the measured intensity values along the same lines of sight. The relative standard deviation (rms) was calculated from the equation

$$
s=\sqrt{\frac{1}{N} \sum_{i=1}^{N}\left[\frac{I_{i}^{\text {data }}-I_{i}^{\text {model }}}{I_{i}^{\text {data }}}\right]}
$$

where $N$ is the number of data points $(=30), I_{i}^{\text {data }}$ is the measured intensity for ith line of sight and $I_{i}^{m o d e l}$ is the corresponding simulated value after scaling. The discretized harmonic model gave the first value for the standard deviation.

Keeping in mind two conditions given above (symmetry and the growth toward the equator), the ionization rate was decreased at the equator $\left(\lambda=0^{\circ}\right)$. If it was found that the standard deviation was less than before, the value at $\lambda=0^{\circ}$ was reduced once again, and the standard deviation was compared to the value achieved during the former trial. If it were greater, the ionization rate was increased. If it were still too large, the same procedure was performed for the values $\lambda= \pm 10^{\circ}$. The procedure was continued until the standard deviation was less than in the case of the discretized harmonic model. Then the ionization rate distribution, which gave a better fit, was taken as a new starting point, and the same procedure was started again from the point $\lambda=0^{\circ}$.

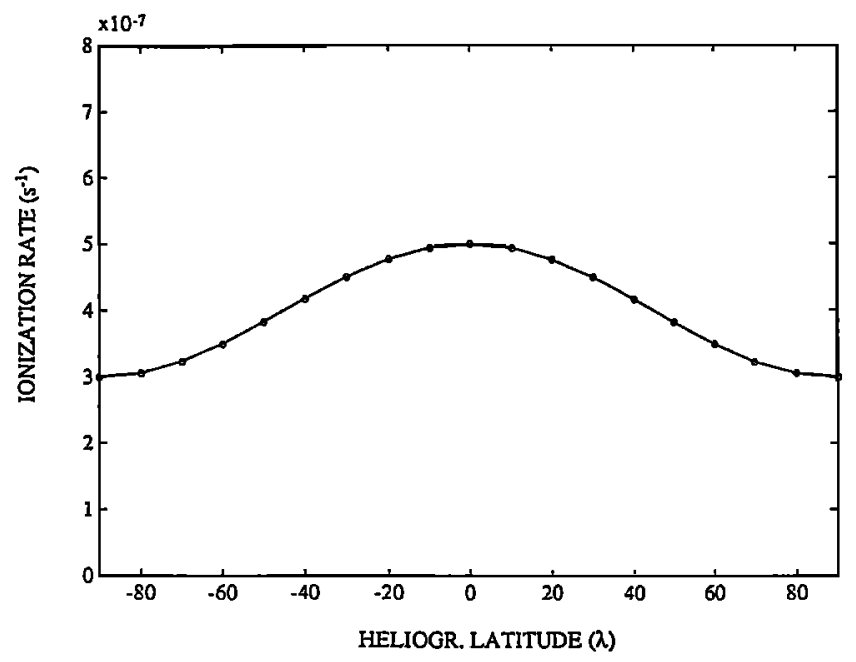

Fig. 2. The discretized harmonic ionization rate, which was used as a starting point in simulations as a function of the heliographic latitude $\lambda$.

\section{MODEL RESULTS}

\subsection{Latitudinal Variation of the Ionization Rate}

The main purpose of the simulations was to find out what kind of latitudinal dependence of the ionization rate would produce the best fit to the data. The final ionization rate distributions for each session are shown in Figure 3 together with the harmonic ionization rate. Figure 4 shows the corresponding intensities and the measured intensity data. A discussion of each session follows and in conclusion their common features are studied. Rms (root mean square) values, densities at infinity, data gaps, and measuring angles $\phi$, which were excluded when calculating the standard deviation for each session, are shown in Table 1.

The different densities at infinity listed in the Table 1 are calculated for the discrete model only. The corresponding values for the harmonic ionization rate range from 0.036 to 0.14 . Thus the relatively good $\mathrm{ms}$ values in the case of the harmonic model does not reflect the whole truth. However, we wanted to compare the best results achieved using two different models.

Since the ecliptic longitude for the session S1 was $11^{\circ}$, the measuring circle covers lines of sights toward both the upwind $\left(\phi \approx 350^{\circ}-20^{\circ}\right.$, see Figure $\left.4 a\right)$ and the downwind direction $(\phi \approx$ $170^{\circ}-200^{\circ}$, see Figure $4 a$ ). The ms value for the discrete model is the worst of all sessions, and there is a visible difference between the data and the model around $\phi=0^{\circ}-70^{\circ}$ (see Figure 4a). Thus this difference cannot be removed through simply changing the ionization rate. However, we do not exclude the possibility, that changing other parameters (such as velocity and temperature) would produce a better result. The multiple scattering is hardly responsible for these discrepancies, since according to Keller et al. [1981], the difference of the intensity between the upwind and downwind direction is overestimated in the optically thin model that we have used. As shown in Figure $4 a$, the difference between the upwind and the downwind direction is underestimated by the model. It is also worth remembering that the velocity of the interstellar gas changes while crossing the heliopause, and its effects are difficult to predict. 
TABLE 1. Rms Values, Densities at Infinity, Data Gaps, and Angles $\phi$ That Were Excluded in the Standard Deviation Calculations for Each Session

\begin{tabular}{|c|c|c|c|c|c|}
\hline Session & $\begin{array}{c}\text { Rms } \\
\text { Discrete model }\end{array}$ & $\begin{array}{c}\text { Rms } \\
\text { Harmonic model }\end{array}$ & $\begin{array}{l}\text { Density at } \\
\text { Infinity }\end{array}$ & Data Gaps & $\begin{array}{c}\text { Excluded Angles } \\
\phi\end{array}$ \\
\hline \multirow[t]{3}{*}{ s1 } & $4.41 \%$ & $4.77 \%$ & $0.036 \mathrm{~cm}^{-3}$ & $184^{\circ}-188^{\circ}$ & \\
\hline & & & & $220^{\circ}-238^{\circ}$ & $228^{\circ}$ \\
\hline & & & & $254^{\circ}-268^{\circ}$ & $264^{\circ}$ \\
\hline \multirow[t]{2}{*}{ s2 } & $2.96 \%$ & $5.62 \%$ & $0.029 \mathrm{~cm}^{-3}$ & $220^{\circ}-252^{\circ}$ & $228^{\circ}, 240^{\circ}, 252^{\circ}$ \\
\hline & & & & $300^{\circ}-326^{\circ}$ & $300^{\circ}, 312^{\circ}, 324^{\circ}$ \\
\hline \multirow[t]{3}{*}{ s3 } & $2.51 \%$ & $3.58 \%$ & $0.034 \mathrm{~cm}^{-3}$ & $224^{\circ}-252^{\circ}$ & $228^{\circ}, 240^{\circ}, 252^{\circ}$ \\
\hline & & & & $284^{\circ}-358^{\circ}$ & $288^{\circ}, 300^{\circ}, 312^{\circ}$ \\
\hline & & & & & $324^{\circ}, 336^{\circ}, 348^{\circ}$ \\
\hline \multirow[t]{7}{*}{ s4 } & $2.61 \%$ & $3.53 \%$ & $0.037 \mathrm{~cm}^{-3}$ & $94^{\circ}-100^{\circ}$ & \\
\hline & & & & $120^{\circ}-130^{\circ}$ & $120^{\circ}$ \\
\hline & & & & $238^{\circ}-278^{\circ}$ & $240^{\circ}, 252^{\circ}, 264^{\circ}$ \\
\hline & & & & & $276^{\circ}$ \\
\hline & & & & $296^{\circ}-348^{\circ}$ & $300^{\circ}, 312^{\circ}, 324^{\circ}$ \\
\hline & & & & & $336^{\circ}$ \\
\hline & & & & $358^{\circ}$ & $348^{\circ}$ \\
\hline
\end{tabular}

TABLE 2. Parameter Values of the Interstellar Wind Used in Simulations

\begin{tabular}{cc}
\hline \multicolumn{2}{c}{ Simulations } \\
\hline Parameter & Values \\
\hline $\mathrm{T}_{\infty}$ & $8000 \mathrm{~K}$ \\
$\mu$ & 0.75 \\
$w_{\text {lon }}$ & $254.5^{\circ}$ \\
$w_{\text {lat }}$ & $6.0^{\circ}$ \\
$v_{0}$ & $20 \mathrm{~km} / \mathrm{s}$
\end{tabular}

Values are taken from Bertawx et al. [1985] and Dalaudier et al. [1984]. $T_{\infty}$ is the temperature of the hydrogen gas at infinity, $\mu$ is the radiation pressure divided by the gravitational force, $v_{0}$ is the bulk velocity of the interstellar gas, and the wind directions $w_{10 n}$ and $w_{1 a t}$ are the longitudinal and the latitudinal angles in the ecliptic coordinate system.

The model results fit the intensity data well in the session S2. Six angles had to be excluded because of data gaps when calculating rms values. This could be the reason for the most striking feature in the discrete ionization rate distribution: the sudden decrease of the ionization rate near the poles. The rms value for the distribution, where the ionization rate at the poles is assumed to be the same as at $\lambda= \pm 80^{\circ}\left(2.6 \times 10^{-7} \mathrm{~s}^{-1}\right.$ in session S2), is $3.00 \%$. Thus the difference of the rms values is less than $0.05 \%$, and the deep slope near the poles is presumably an artificial feature, which reflects the great uncertainty of the result near the poles. In session S3 the fitting is mostly based on the data from the first half of the measuring cycle, since there are two large data gaps around $\phi=$ $224^{\circ}-252^{\circ}$ and $\phi=284^{\circ}-358^{\circ}$.

The discrete ionization rate for the session S4 greatly resembles the ionization rate for the session $\mathbf{S 2}$. S4 and S2 share a sharp structure at the equator and a deep slope toward the zero at the poles. Because of these deep slopes, there are two sharp local maximum points in the intensity figure near $\phi=80^{\circ}$ and $\phi=260^{\circ}$. If we straighten these slopes, the rms value would be $2.71 \%$, and the sharp structures near the local maxima would disappear.

In each session there are two similar structures present. First, there is an area of enhanced ionization near the equator. This area covers the region from $\lambda=-30^{\circ}$ to $\lambda=30^{\circ}$. Second, there are two broad plateaus from $\lambda= \pm 30^{\circ}$ to $\pm 70^{\circ}$. These phenomena are quite possible if one considers the coronal structure of the Sun. According to solar observations, during the solar minimum, the large polar coronal holes reach to the low heliographic latitudes, and there is a narrow belt of slow solar wind, which lies along the equator. These phenomena seem to indicate more rapid change in the ionization rate near the equator, while the harmonic model predicts slower change. On the other hand, the increase of the ionization toward the equator was already explained by the harmonic model.

Hydrogen atoms that scatter solar Lyman alpha radiation are exposed to the solar wind for several years, since their velocity is only $4 \mathrm{AU}$ in a year. The main contribution of the measured intensity along the line of sight comes from the distance 5-10 AU that is, from the atoms, which have been influenced by the solar wind during the last 2-3 years. Thus the best ionization rate distribution is a kind of average over ionizing effects of the Sun during the last two or three years. This is probably why the enhanced ionization near the equator is in as broad a range 

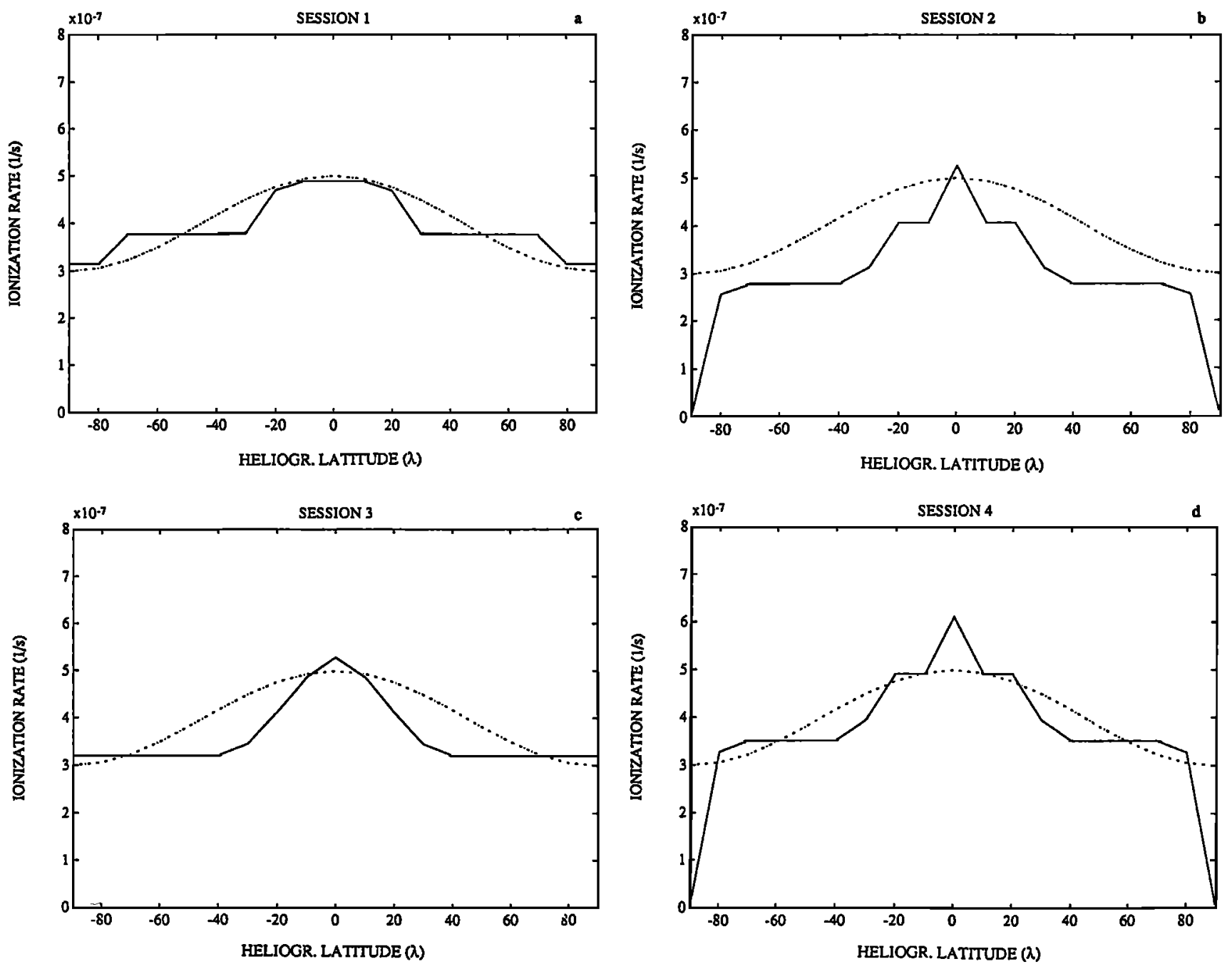

Fig. 3. The discrete ionization rate (solid line) and the harmonic ionization rate (dashed line) as a function of the heliographic latitude $\lambda$ for the session (a) S1, (b) S2, (c) S3, and (d) S4.

as $-30^{\circ}$ to $30^{\circ}$. The reason then for broad plateaus are the coronal holes. The mean ionization rate calculated from four sessions is shown in Figure 5. The common features explained above are clearly visible.

All results given above are calculated using fixed values of the interstellar wind parameters given in Table 2. Since there are so many variable parameters, the use of any other method based on the inversion theory was impossible. Also, the effect of varying the parameters could not be thoroughly tested. The basic reason for this was the limited CPU time. Ionization rate calculations required 300-1300 trials, until the best model was found.

However, a few test runs were performed for the session S3. It was chosen for its smooth latitudinal variations. The ionization rate for the session $\mathbf{S 3}$ shown in Figure $3 c$ was used as a starting point in these checking calculations. We changed the interstellar wind parameters, within the limits of error given by Lallement et al. [1985b] except the bulk velocity, which was changed by $\pm 2 \mathrm{~km} / \mathrm{s}$. In checking runs, only one parameter was changed, while other parameters were kept fixed.

In most of the test runs, the ionization rate changed very little. As a typical example of the changes, the ionization rate, when the temperature $T$ is $10000 \mathrm{~K}$, is shown in Figure 6 . Also, in the cases when $T=6000 \mathrm{~K}, \mu=0.65, \mu=0.85$, the longitude of the wind in ecliptic coordinates is $253^{\circ}$, the latitude is $4.5^{\circ}$, the bulk velocity $v$ is $18 \mathrm{~km} / \mathrm{s}$ or $v$ is 22 $\mathrm{km} / \mathrm{s}$, the ionization rate deviated only slightly. However, when the longitude of the wind in ecliptic coordinates is $256^{\circ}$ or the latitude is $7.5^{\circ}$, the level of the ionization rate changed considerably. As an example, the case when the latitude is $7.5^{\circ}$ is shown in Figure 7. Thus the level of the ionization rate seems to vary when the interstellar parameters are changed, but the common features, the enhanced ionization rate at the equator and the plateaus at $\pm 30^{\circ}$ to $\pm 70^{\circ}$, remain unchanged in. each of the test runs.

\subsection{Estimated Latitudinal Variation of the Solar Wind Flua}

If the ionization rate as a function of the heliographic latitude is known, the latitudinal variation of the solar wind proton flux (or solar wind proton density) can be calculated from (3). As an example of the derivation of the solar wind proton flux, the result of the session $\mathrm{S} 3$ is considered below. Assuming

$$
n_{p x}(r, \lambda)=n_{e}^{p r}(\lambda) \frac{r_{e}^{2}}{r^{2}}
$$



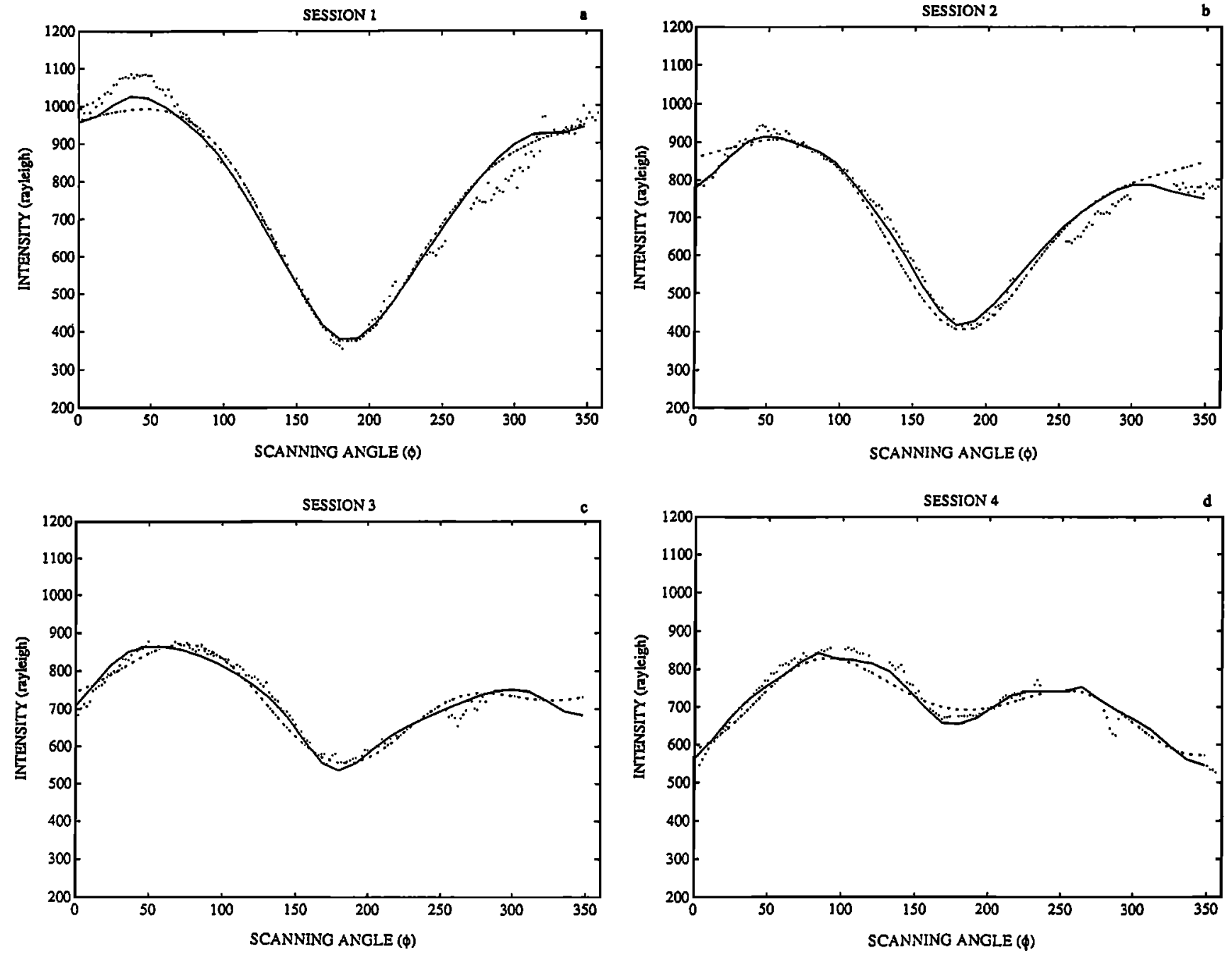

Fig. 4. The intensity data measured during the session (a) S1, (b) S2, (c) S3, and (d) S4 (dots) and calculated intensities using the discrete ionization rate shown in Figure 3 (solid line) and the harmonic ionization rate (dashed line) as a function of the scanning angle $\phi$.

and $v_{\mathrm{rel}}(\lambda, r)=\mathbf{v}_{\mathrm{pr}}(\lambda, r)=\mathbf{v}_{\mathrm{pr}}(\lambda)$, we have

$$
\beta\left(\lambda_{r} r\right)=n_{e}^{p r}(\lambda) \frac{r_{e}^{2}}{r^{2}}\left|v_{p r}(\lambda)\right| \sigma_{c e}\left(\left|v_{p r}(\lambda)\right|\right)+\beta_{p h}(\lambda, r)
$$

For the cross section an experimental expression by Fite et al. [1962],

$$
\sqrt{\sigma_{c e}}=7.6 \times 10^{-8}-1.06 \times 10^{-8} \log _{10} \mathrm{E}
$$

is used. The cross section is given in units of square centimetres, and the energy of a proton, $E$, is expressed in electron volts.

Kojime and Kakinuma [1990] have reviewed latitudinal dependence of the solar wind as deduced from IPS measurements and spacecraft measurements. They have listed three works, which concem the latitudinal dependence of the solar wind in 1976 and 1977. Here the results from Zhao and Hundhausen [1981] (model 4 of Kojima and Kakinuma [1990]) are used. According to them there was a relationship between the solar wind speed and the angular displacement $\lambda$ from the neutral sheet. The relationship in 1976 was estimated to be (see Figure 8)

$$
\begin{gathered}
v_{\mathrm{pr}}=350 \mathrm{~km} \mathrm{~s}^{-1}+800 \sin ^{2} \lambda \mathrm{km} \mathrm{s}^{-1},|\lambda| \leq 35^{\circ} \\
v_{\mathrm{pr}}=600 \mathrm{~km} \mathrm{~s}^{-1},|\lambda|>35^{\circ}
\end{gathered}
$$

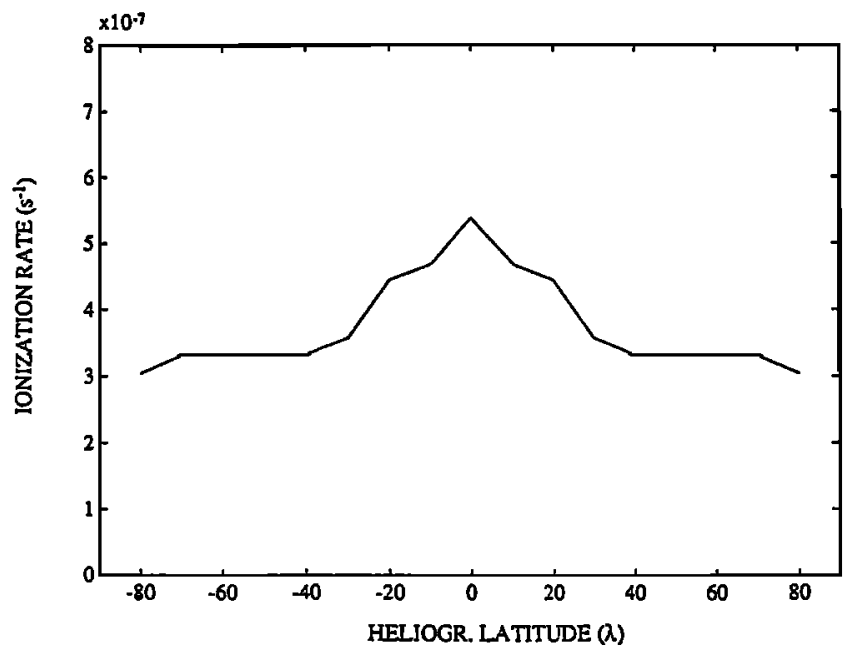

Fig. 5. The mean ionization rate calculated from four sessions S1, S2, S3, and S4 as a function of the heliographic latitude $\lambda$. 


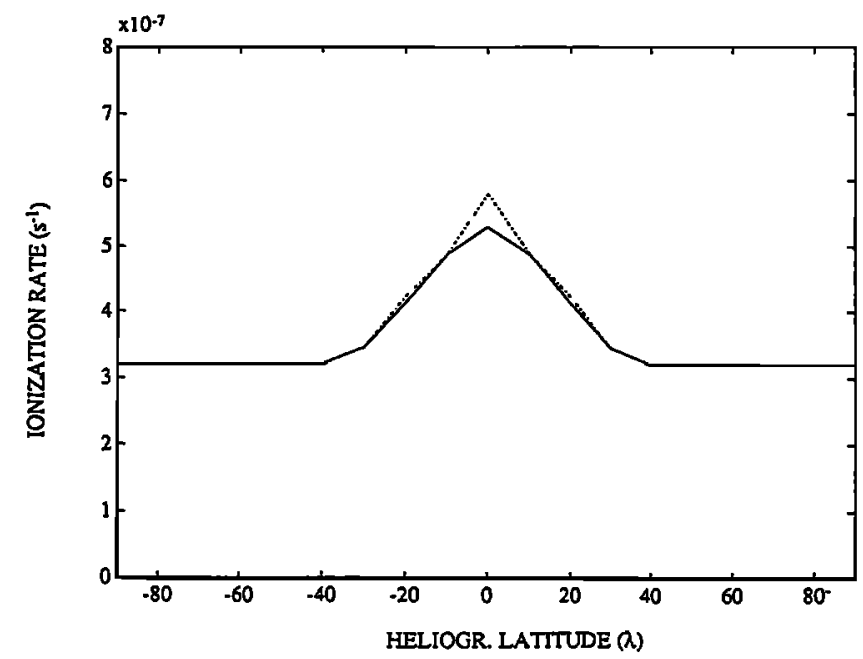

Fig. 6. The ionization rate as a function of the heliographic latitude $\lambda$ for the session $S 3$ (dashed line), when the parameters are $T=10000 \mathrm{~K}, \mu=0.75$, the longitude of the wind in ecliptic coordinates is $254,5^{\circ}$, the latitude is $6.0^{\circ}$ and $v=20 \mathrm{~km} / \mathrm{s}$. The ionization rate, when $\mathrm{T}=8000 \mathrm{~K}$ (solid line), respectively.

In this work it is assumed, that the angular displacement from the neutral sheet is approximately the same as the heliographic latitude, also marked with $\lambda$. The corresponding cross sections range from $\sigma_{\mathrm{ce}}(350 \mathrm{~km} \mathrm{~s})=2.140 \times 10^{-15} \mathrm{~cm}^{2}$ to $\sigma_{\mathrm{ce}}\left(600 \mathrm{~km} \mathrm{~s}^{-1}\right)=1.705 \times 10^{-15} \mathrm{~cm}^{2}$.

Using (16), (17), and (18), the approximate solar wind proton flux $n_{p r}(\lambda, r) \mid v_{r e l}(\lambda, r)$ and the solar wind proton density can be solved. These quantities for the session $\mathbf{S 3}$ are shown in Figure 9. The photoionization is assumed to be $0.88 \times 10^{-7} \mathrm{~s}^{-1}$.

As shown in Figure 9, the solar wind proton flux normalized to 1 AU grows from $1.36 \times 10^{8} \mathrm{~cm}^{-2} \mathrm{~s}^{-1}$ at the poles to $2.06 \times 10^{8} \mathrm{~cm}^{-2} \mathrm{~s}^{-1}$ at the equator, that is, a growth of $34 \%$ in the solar wind proton flux toward the equator. This is in agreement with the values $25.35 \%$ given by Lallement et al. [1985b] using the harmonic model.

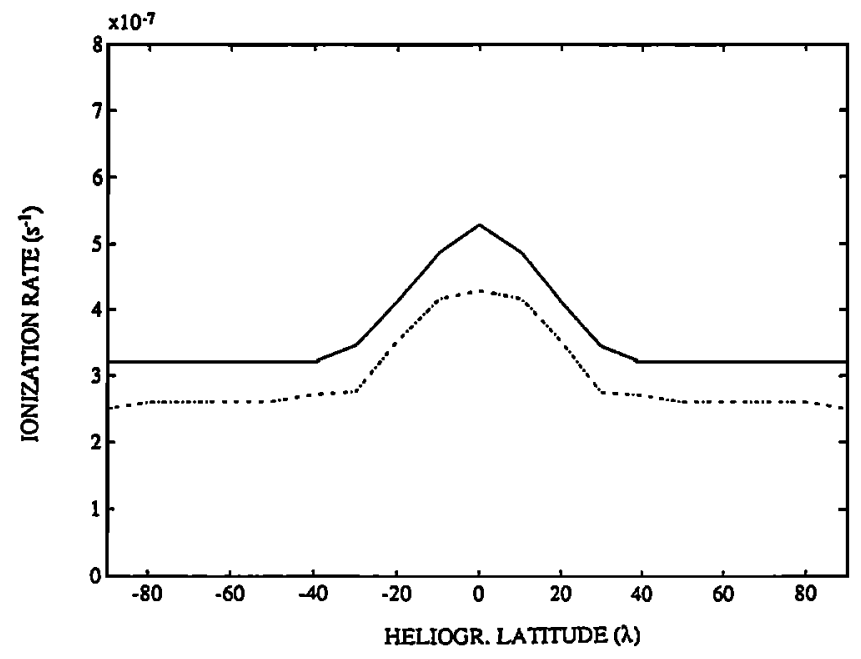

Fig. 7. The ionization rate as a function of the heliographic latitude $\lambda$ for the session $S 3$ (dashed line), when the parameters are $\mathrm{T}=8000 \mathrm{~K}, \mu=0.75$, the longitude of the wind in ecliptic coordinates is $254,5^{\circ}$, the latitude $7.5^{\circ}$ and $v=20 \mathrm{~km} / \mathrm{s}$. The ionization rate, when the latitude is $6.0^{\circ}$ (solid line), respectively.

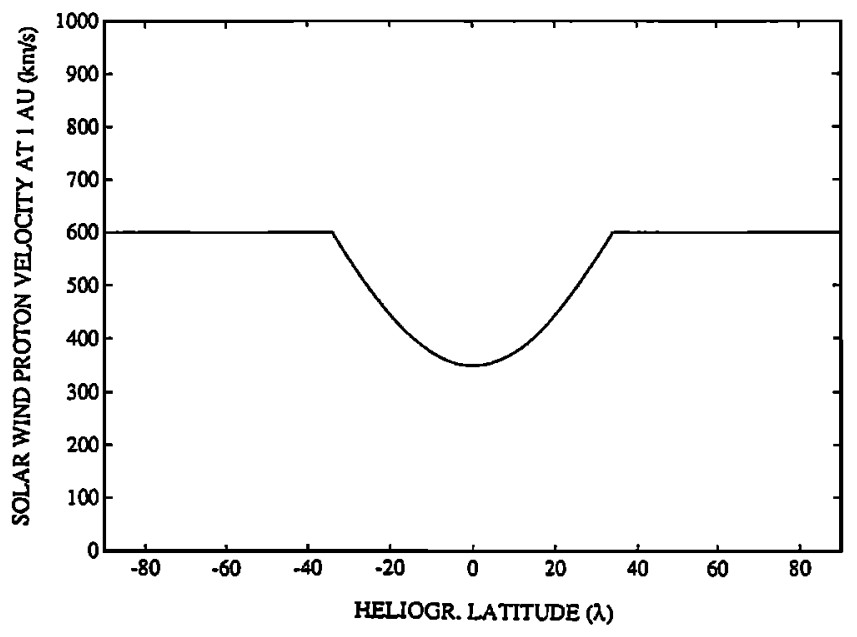

Fig. 8. The latitudinal dependence of the solar wind velocity as a function of the heliographic latitude $\lambda$ in 1976 according to Zhao and Hundhausen [1981].
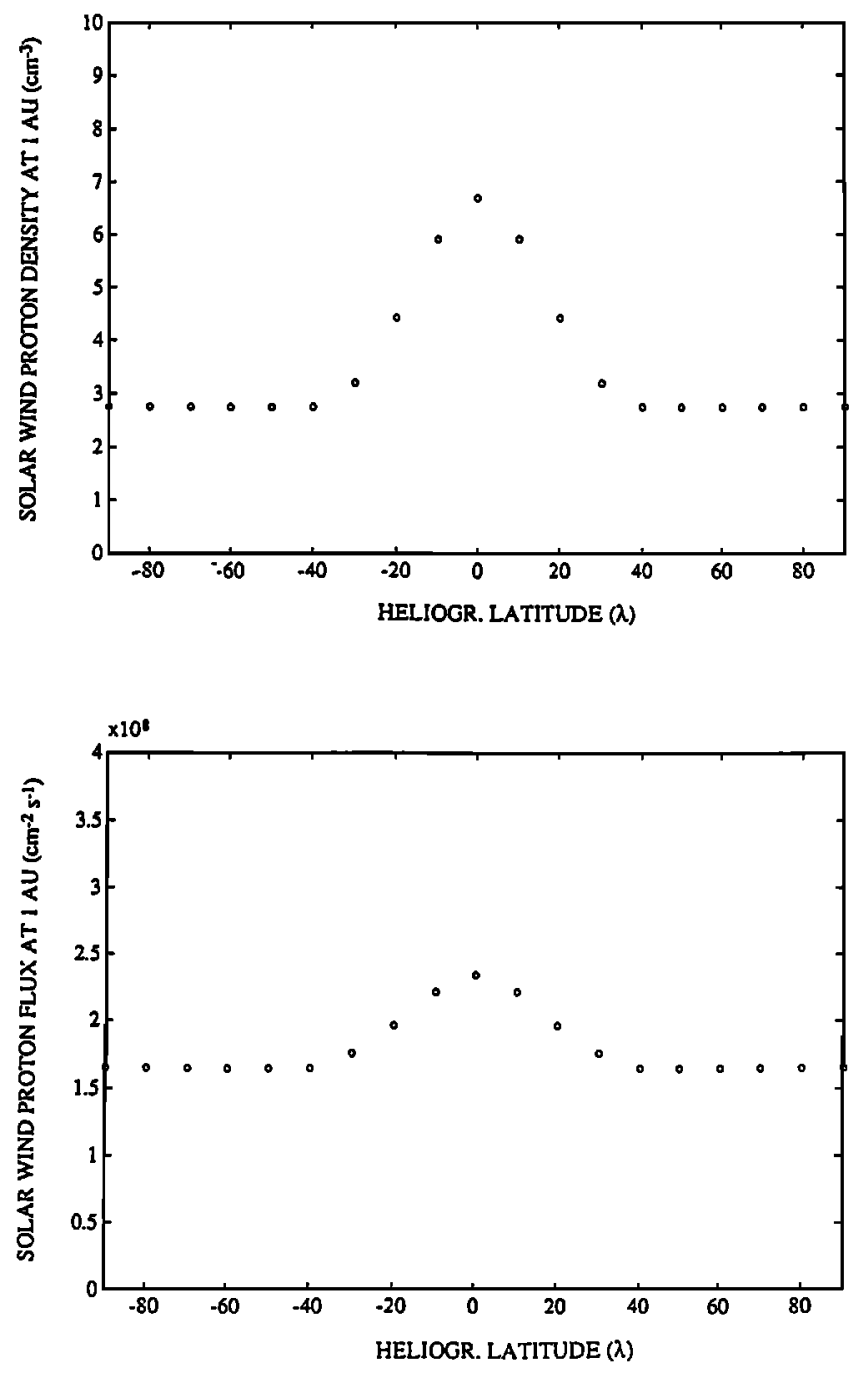

Fig. 9. The latitudinal dependence of the solar wind proton flux and density as a function of the heliographic latitude $\lambda$ as deduced from discrete ionization rate distribution for the session S3. 
However, it is worth noticing that the prediction about the solar wind proton flux is strongly dependent on the solar wind proton velocity model used. If two other models for the year 1976 reviewed by Kojima and Kakinuma (models 5 and 6 used by Kojima and Kakinuma [1990, Table 1]) were used, the change in the solar wind proton flux at $1 \mathrm{AU}$ would be $26 \%$ and $36-40 \%$ (different values in the velocity model for the north and the south poles), respectively. The first of these two velocity models, model 5 , is based on spacecraft observations, that were made in a narrow latitudinal range near the solar equator. Thus the plateaus at high latitudes are missing. The second velocity model, model 6 , has lower plateaus than model 4 in Figure 8 . In fact, both models 4 and 6 have quite smooth latitudinal gradient, IPS measurements during the next solar cycle would prefer sharper gradient. These older models were derived from the IPS data without considering the integration effect caused by the line-of-sight integrals, as pointed out by Kojima and Kakinuma. However, in lack of better data, model 4 is used, since the plateaus are more pronounced there.

When the change of the solar wind proton flux from the plateaus to the equator is calculated using the ionization rate of another session, the session S4, the growth is about $28 \%$. Thus the prediction by Lallement et al. (1985b) seems to be reasonable.

Recently, Yang and Schunk [1991] have studied theoretically the latitudinal dynamics of steady solar wind flows beyond $0.15 \mathrm{AU}$. In their MHD simulations they have calculated the latitudinal structure of the solar wind. Their results indicate the existence of a proton number density maximum at the magnetic neutral line.

\section{Discussion and Conclusions}

The main results of this work are the following. First, it has been shown that the intensities derived from the model best fit the intensity data when the harmonic ionization rate is replaced with a new distribution, where the enhanced ionization near the solar equator and large plateaus around latitudes $\pm 30^{\circ}- \pm 70^{\circ}$ are present. Second, it has been shown that the new ionization rate distributions derived using the intensity data predicts $25-35 \%$ growth of the solar wind mass flux from the polar areas toward the equator as proposed by Lallement et al. [1985b].

The deep slopes of the ionization rate toward zero near the poles that appear in the results of sessions S2 and S4 are very likely artificial features. In fact, negative values of the ionization rate at $\lambda= \pm 90^{\circ}$, would give still better rms values. However, any reliable physical model for the Sun would not produce negative ionization rate values. Thus the values of the ionization rates obtained here, which are around $\pm 90^{\circ}$ to $\pm 70^{\circ}$, are highly questionable.

Although there is only a small difference in the rms values of the calculated intensities between the harmonic model and the discrete models, there is a clear difference between the ionization rates. Also, it is shown that the best $\mathrm{mms}$ values, which can be achieved using the symmetric ionization rate, are of the order $2.5 \%$. This reflects partly the uncertainty of the method used to remove the contribution of the geocorona and partly, perhaps, the uncertain values of parameters. The geocorona had a considerable effect on data measured during the latter half cycle of the measuring circle.

The method that we have used is not precise enough to predict the exact dependence of the solar wind proton flux on the latitude. Because of the limited amount of computer time, the method could not be checked thoroughly. Also, the available latitudinal resolution is poor especially at high heliographic latitudes. Therefore the results near the poles and the width of the plateaus are questionable. One has to keep in mind that these solutions of this ill-conditioned problem are not necessarily the only possible solutions, and the method could have produced biased effects.

Also, when deriving the formulas for the density distribution and the optical model, several assumptions were made, the validity of which can be questioned. First, the flow of the interstellar gas was considered as a stationary problem, although the solar wind and the EUV radiation are strongly dependent on time [see Ajello et al., 1987]. This presumption was used when the equation of continuity was solved and when the ratio of the force on an atom due to radiation pressure to that of gravity was assumed to be constant. Thus 27 day variations caused by the rotation of the Sun as well as 11-year cycles were neglected.

In the hot model it was assumed that the velocity distribution at infinity is Maxwellian; that is, the gas is in thermal equilibrium. To approach equilibrium, the nonuniformities of the temperature, density, and average velocity have to be smoothed out through collisions from one part of the gas to another. The average distance over which properties of the gas can be transported in one elastic collision; that is, the mean free path is approximately $10^{17} \mathrm{~m}$. It is of the order of parsecs as the small structure of a diffuse interstellar cloud as given by McKee and Ostriker [1977]. However, the thermalization of neutral gas is possible also through elastic and charge exchange collisions with other particle species. Thus the Maxwellian distribution is reasonable.

It is also worth noticing that multiple scattering effects change the Lyman $\alpha$ intensity distribution. The assumption of the optically thin medium underestimates the radial sky background intensity by $5-35 \%$ depending upon the angle between the interstellar wind and the line of sight [see Keller et al., 1981]. The study of this phenomenon in the case of Prognoz data is in progress. The presumption concerning the shape of the solar Lyman a line have certainly visible effects on the results. On the other hand, only a part of the information is retained in the line-of-sight integration in the intensity measurements (see section 3 ). Thus the results are always averages over a long time (2-3 years) and distance (1-12 AU).

Many minor effects were ignored too. For example, only the effect of the solar wind protons was taken into account, while all other species were forgotten. Also, the $r^{-2}$ dependence in the ionization rate was retained, fast neutrals were neglected, and finally, all the effects of the heliopause were disregarded.

If the interstellar parameters could be derived independently and a large set of Lyman $\alpha$ data were available, the method could be tested more thoroughly. Fortunately, this will come true in 1995, when the SWAN (Solar Wind ANisotropies) experiment onboard the SOHO satellite will start to measure Lyman $\alpha$ data. At the same time, Ulysses will be measuring the solar wind. Then Ulysses in situ helium measurements will give data concerning the interstellar wind parameters simultaneously with the solar wind experiments of the velocity and the density of the solar wind. Furthermore, the WIND mission will provide baseline ecliptic plane observations while Ulysses and SOHO observe the Sun. WIND will be 
launched in summer 1993. Then the full testing of this method will be possible.

Finally, the ionization rate distribution proposed above, is probably the furthest limit, which can be achieved using a stationary model. More precise results require the use of a timedependent model including multiple scattering effects, which is the next step in modeling the flow of the interstellar matter into the heliosphere.

Acknowledgments. We would like to thank the referees for the careful examination of the manuscript and very useful comments.

The Editor thanks $\mathrm{K}$. Richter and D. Judge for their assistance in evaluating this paper.

\section{REFERENCES}

Ajello, J. M., A. I. Stewart, G. E. Thomas, and A. Graps, Solar cycle study of interplanetary Lyman-alpha variations: Pioneet Venus orbiter sky background results, Astrophys. J., 317, 964, 1987.

Axford, W. I., The interaction of the solar wind with the interstellar medium, in Solar wind, edited by C. P. Sonett, P. J. Coleman, Jr., and J. M. Wilcox, NASA Spec. Publ. SP-308, 609, 1972.

Axford, W. I., The solar wind, Sol. Phys., 100, 575, 1985.

Baranov, V. B., Gas dynamics of the solar wind interaction with the interstellar medium, Space Sci. Rev, 52, 89, 1990.

Bertaux, J. L., R. Lallement, V. G. Kurt, and E. N. Mironova, Characteristics of the local interstellar hydrogen determined from Prognoz 5 and 6 interplanetary Lyman $\alpha$ profile measurements with a hydrogen absorption cell, Astron. Astrophys., 150, 1, 1985.

Blum, P. W., and H. J. Fahr, Interaction between interstellar hydrogen and the solar wind, Astron. Astrophys., 4, 280, 1970.

Brandt, J. C., and Chamberlain, J. W., Interplanetary gas, I, Hydrogen radiation in the night sky, Astrophys. J., 130,670, 1959.

Chamberlain, J. W., Calculation of polarization and anisotropy of resonant and fluorescent scattering, Icarus, 84, 106, 1990.

Chamberlain, J. W., and D. M. Hunten, Theory of Planetary Atmospheres, An Introduction to Their Physics and Chemistry, Pp. 290302, 2nd ed., Academic, San Diego, Calif., 1987.

Coles, W. A., and B. J. Rickett, IPS observations of the solar wind speed out of the ecliptic, J. Geophys. Res., 81, 4797, 1976.

Dalaudier, F., J. L. Bertaux, V. G. Kurt, and E. N. Mironova, Characteristics of interstellar helium observed with Prognoz 6 58.4-nm photometers, Astron. Astrophys., 134, 171, 1984.

Fahr, H. J., On the influence of neutral interstellar matter on the upper atmosphere, Astphys. Space Sci., 2, 474, 1968.

Holzer, T. E., Interaction between the solar wind and the interstellar medium, Ann. Rev. Astron. Astrophys., 27, 199, 1989.

Isenberg, P. A., and B. H. Levy, Polar enhancements of interplanetary La through solar wind asymmetries, Astrophys. J., 219, L59, 1978.

Johnson, H. E., Backscatter of solar resonance radiation, I, Planet. Space Sci., 20, 829, 1972.

Joselyn, J. A., and T. E. Holzer, The effect of asymmetric solar wind on the Lyman $\alpha$ sky beckground, J. Geophys. Res. 80, 903, 1975.

Keller, H. U., K. Richter, and G. E. Thomas, Multiple scattering of solar resonance radiation in the nearby interstellar medium, II, Astron. Astrophys., 102, 415, 1981.
Kojima, M., and T. Kakinuma, Solar wind speed, Space Sci. Rev., 53 , $173,1990$.

Kumar, S., and A. L. Broadfoot, Evidence from Mariner 10 of solar wind flux depletion at high ecliptic latitudes, Astron. Astrophys., 69, L5-L8, 1978.

Kumar, S., and A. L. Broadfoo, Signatures of solar wind latitudinal structure in interplanetary Lyman $\alpha$ emissions: Mariner 10 observations, Astrophys. J., 228, 302, 1979.

Lallement, R., J. L. Bertaux, V. G. Kurt, and E. N. Mironova, Observed perturbations of the velocity distribution of interstellar hydrogen atoms in the solar system with Prognoz Lyman alpha measurements, Astron. Astrophys, 140, 243, 1984.

Lallement R., J. L. Bertaux, and F. Dalaudier, Interplanetary Lyman $\alpha$ spectral profiles and intensities for both repulsive and attractive solar force fields: Predicted absorption pattem by a hydrogen cell, Astron. Astrophys., 150, 21, 1985 a.

Lallement, R., J. L. Bertaux, and V. G. Kurt, Solar wind decrease at high heliographic latitudes detected from Prognoz interplanetary Lyman alpha mapping, J. Geophys. Res., 90, 1413, 19856.

Lee, M. A., The solar wind terminal shock and the heliosphere beyond, in Solar Wind Six Tech. Note, NCAR/TN-306+Proc., 635, Natl. Cent. for Atmos. Res., Boulder, Colo., 1988.

Lemaire, P., J. Charra, A. Jouchoux, A. Vidal-Madjar, G. E. Artzner, J. C. Vial, R. M. Bonnett, and A. Skumanich, Calibrated full disk solar H I Lyman $\alpha$ and Lyman B profiles, Astron. Astrophys., 223, L55, 1978.

McKee, C. F, and J. P. Ostriker, A theory of the interstellar medium: three components regulated by supernova explosions in an inhomogeneous substrate, Astrophys. J., 218, 148, 1977.

Smith, E. J., and A. Bames, Spatial dependences in the distant solar wind: Pioneers 10 and 11, in Solar Wind Five, NASA Conf. Publ. 2280, 521, 1983.

Suess, S. T, The heliopause, Rev. Geophys., 28, 1, 97, 1990.

Summanen, T., Latitudinal distribution of solar wind as deduced from Lyman alpha measurements, Lic. thesis, Univ. of Helsinki, 1992.

Weissbluth, M., Atoms and molecules, p. 516, Academic, San Diego, Calif., 1978.

Witt, N., J. M. Ajello, and P. W. Blum, Solar wind latitudinal variations deduced from Mariner 10 interplanetary $H(1216 \AA)$ observations, Astron. Astrophys., 73, 272, 1979.

Witt, N., J. M. Ajello, and P. W. Blum, Polar solar wind and interstellar wind properties from interplanetary Lyman- $\alpha$ radiation measurements, Astron. Astrophys., 95, 80, 1981.

Yang, W.-H., and R. W. Schunk, Latitudinal dynamics of steady solar wind flows, Astrophys. J., 372, 703, 1991.

Zhao, X.P.. and A. J. Hundhausen, Organization of solar wind plasma properties in a tilted, heliomagnetic coordinate system, $J$. Geophys. Res., 86, 5423, 1981.

J. L. Bertaux and R. Lallement, Service d'Aeronomie du Centre National de la Recherche Scientifique, F-91310 Verrières-le-Buisson, France.

E. Kyrölä and T. Summanen, Finnish Meteorological Institute, P. O. Box 503, 00101 Helsinki, Finland.

(Received August 8, 1992;

revised January 8, 1993;

accepted January 12, 1993.) 